\title{
The two parts of the second law of thermodynamics
}

\author{
Mário J. de Oliveira*10 \\ ${ }^{1}$ Universidade de São Paulo, Instituto de Física, São Paulo, SP, Brasil
}

Received on June 09, 2017; Revised on July 09, 2018; Accepted on July 18, 2018.

\begin{abstract}
According to Sommerfeld, the well known Clausius and Kelvin statements of the second law of thermodynamics comprises two parts. The first part includes the Carnot principle that all Carnot engines operating between the same temperatures have the same efficiency. The second part contains the law of increase in entropy. Usually, the two parts are understood as a logical consequence of these statements, including the Carnot principle. Here, we argue that this principle need not be a derived law and may be considered as a fundamental law, without the need of demonstration. To this end we analyze the roots of the second law, which are contained in the memoir of Carnot on the production of work by heat, and its emergence in the papers of Clausius on heat.
\end{abstract}

Keywords: thermodynamics, second law of thermodynamics, Carnot principle, Clausius principle.

\section{Introduction}

The second law of thermodynamics as conceived by Clausius [1] and Kelvin [2] can be understood as having two parts, which Sommerfeld [3] called the first and second parts of the second law. The first part includes the Carnot principle, which states that all engines operating according to the Carnot cycle with the same temperatures have the same efficiency, and its main consequence that the ratio $d Q / T$ of the infinitesimal heat and the temperature is an exact differential, which allows the definition of entropy. The second part comprises the law of increase in entropy. In addition to the statements of Clausius and Kelvin, several other statements of the second law have appeared. Some are equivalent to the statements of Clausius and Kelvin, some are equivalent to the first part only, and some are equivalent to the second part only.

The first aspect that comes to mind regarding the two parts is that the first part concerns systems that are in thermodynamic equilibrium, or more precisely systems that are undergoing an equilibrium process. The second part, on the other hand, refers to systems that are not in equilibrium. If the second law of thermodynamics is understood as a law about systems undergoing nonequilibrium processes, than only the second part should properly be identified as the second law.

Usually, both the first and the second part of the second law are derived from the Clausius or Kelvin statements of the second law. However, the derivation is not free from difficulties and, in fact, it has been criticized on the grounds that the reasonings and the hidden assumptions employed in the derivation are not totally clear [4]. Since the Carnot principle, of the first part, is considered to

*Correspondence email address: oliveira@if.usp.br be true for systems in thermodynamic equilibrium, these difficulties can be overcome by assuming that the Carnot principle is an independent fundamental law, without the need of demonstration. As to the second part, we will see later that it can be derived from the fundamental law that heat spontaneously flow from a hot to a cold body, which we call Clausius principle.

The independence of the Carnot and Clausius principles, and thus of the two parts of the second law, has conceptually certain advantages. Temperature is considered to be an elementary concept of the theory and entropy is a derived concept defined by $d S=d Q / T$. One could invert this proposition and assume that entropy is an elementary concept, and define temperature from entropy, for systems in equilibrium. In fact, this is the point of view implicit in the Gibbs approach to equilibrium thermodynamics [5,6]. Clausius principle is then used to show that entropy has the properties of convexity, which is the hallmark of Gibbs equilibrium thermodynamics. This is also the point of view adopted by Callen in his Thermodynamics [7].

Another advantage is found in the development of a thermodynamics for systems out of equilibrium. Considering Carnot principle as independent of Clausius principle, we may dispose of the Carnot principle, which is valid for systems in equilibrium, without affecting Clausius principle which continues to be valid. We should also mention the simplification one gains by teaching the fundamental laws of thermodynamics according to the present perspective 8 .

From the theoretical point of view, the roots of the second law, and of the Carnot and Clausius principles, are found on the memoirs of Carnot 99 and Clapeyron [10]. The emergence of the second law is found in the first paper by Clausius on heat [1. Although the memoirs 
of Carnot and Clapeyron did not receive a proper attention of the scientific community at the time of their publications [11, in 1824 and 1834, respectively, they were recognized later on by Kelvin in 1848 [12 and by Clausius in 1850 [1]. In the following, we present the theoretical development of the theory of heat in the period around the transition from the caloric theory to thermodynamics, focusing on the papers of Carnot and Clausius on heat. At the same time we present our point of view concerning the two parts of the second law of thermodynamics.

\section{Carnot's theory}

The investigation on the theory of heat before the emergence of thermodynamics comprised the search for laws related to the thermal behavior of bodies, such as the adiabatic and thermal expansion of gases, and the determination of the specific heat of the substances 13,14 . The production of mechanical work by heat was a subject that was not addressed from a general theoretical point of view, despite the fact that it was quite evident that a gas performs work in an expansion and that the steam machine, which produces mechanical work from heat, had already been invented and developed since the beginning of the eighteenth century [15. A notable exception was Carnot who published in 1824 a book on the subject, entitled Réflexions sur la Puissance Motrice $d u$ Feu [9], that is, thoughts on the mechanical work produced by heat. He was followed on this matter by Clapeyron, who published in 1834 a memoir where he presented Carnot's ideas in analytical form by the use of the pressure-volume diagram, a convenient expedient since work is identified as an area in this diagram [10]. The publications of Carnot and Clapeyron, which revealed to be relevant for the developement of thermodynamics, received little attention [11 but were finally recognized and appreciated by Kelvin in 1848 [12] and by Clausius in 1850 [1.

The relationship between mechanical work and heat was approached by Carnot within the prevailing theory of heat of his time, the caloric theory [13, 14], which recognizes heat as a material fluid called caloric. According to Poisson [16] caloric is an imponderable material substance contained in every part of a body that passes from one body to the other without being annihilated or created. The conservation of caloric in thermal processes was expressed as a law by Lavoisier and Laplace in their memoir on heat as follows 17:

Proposition 1: the amount of free heat always remains the same in the simple mixture of bodies.

In addition to the conservation of caloric, which Carnot took for granted, he grounded his theory on a fundamental rule concerning the behavior of the caloric. According to this rule 9 ,

\begin{abstract}
Proposition 2: caloric flows in such a way as to restore equilibrium, passing from a hot body to a cold body.
\end{abstract}

Equilibrium of the caloric is reached when the temperatures of the bodies become equal. The production of work in a heat machine, explains Carnot, is due to the transport of caloric from a hot to a cold body, and not to its consumption. A consequence of this fundamental rule is that a necessary condition for the production of work by a heat machine is the existence of bodies at distinct temperatures inducing a flow of caloric. How the flow of caloric produces mechanical work was explained by Carnot by an ingenious manner as we shall see.

A keen observation of Carnot concerning the functioning of a heat machine is its cyclic operation. The agent that produces works, which may be a gas, a vapor or any other substance susceptible of volume changes, operates in a cycle, absorbing heat from a reservoir at a higher temperature and delivering heat to a reservoir at a lower temperature. Carnot makes use of a specific cycle, consisting of four successive stages. (1) In the first, the working agent undergoes an isothermal expansion while being in contact with a heat reservoir at a temperature $\theta_{1}$. (2) In the second, kept in isolation, the working agent undergoes a volume expansion and a consequent fall of temperature. (3) In the third, the working agent undergoes an isothermal compression while being in contact with a heat reservoir at a temperature $\theta_{2}$ smaller than $\theta_{1}$. (4) In the last stage, the working agent in isolation undergoes a volume contraction and a consequent rise of temperature. In stage (1) the working agent receives a certain quantity of heat $q$ from the reservoir, whereas in stage (3) it delivers the same quantity of heat $q$ to the reservoir, in accordance with the law of conservation of caloric. In stages (2) and (4) there is no exchange of caloric between the working agent and the environment.

Carnot asks for the condition for the maximum work one can extract from a heat machine in regard to a given quantity of heat. This should occur if any change in temperature of the working agent is accompanied by the change of its volume, and a consequent production of work. If this does not happen, the flow of caloric, due to the difference in temperatures, would not produce work and would be a useless reestablishment of the equilibrium of the caloric, that is, a direct passage of the caloric from a hot to a cold body without any other effect. To avoid the direct passage of caloric, the working agent should not be in contact with any other body. That is the reason why in the processes (2) and (4) of the cycle the working agent is in isolation, meaning that there is no exchange of heat. Carnot explains that this type of process is the same as that considered by Laplace to explain the propagation of sound in air [18, and occurs in a rapid expansion or a sudden contraction of a gas enclosed on a recipient.

According to our understanding, each stage of the cycle is carried out in such a way that the working agent undergoes an equilibrium process. This understanding is 
implicit in the Clapeyron presentation of Carnot cycle due to his use of the pressure-volume diagram [10]. Any point of this diagram is understood as an equilibrium state and a trajectory drawn on it is understood as a sequence of equilibrium states, that is, an equilibrium process.

In the following, Carnot argues that the work does not depend on the substance of the working agent. This fundamental principle is expressed by Carnot as follows 9]:

Proposition 3: The motive power of heat is independent of the agents used to realize it; its quantity is fixed solely by the temperatures of the bodies between which, finally, the transport of caloric is effected.

It is implicit in this statement that it refers to the Carnot cycle described above.

To explain how work is actually generated in a heat machine operating according to his cycle, Carnot makes a surprising and striking analogy with a mechanical system. When a load descends from a certain height to a lower height, it performs a quantity of work which is proportional to the mass of the load and to the difference in heights. Thus, when a certain quantity of heat $q$ "descends" from a high temperature $\theta_{1}$ to a low temperature $\theta_{2}$, the working agent performs a mechanical work $w$ which is proportional to $q$ and to the difference in temperatures $\theta_{1}-\theta_{2}$. According to the fundamental principle, proposition $3, w$ does not depend on the substance of the working agent. Carnot does not say explicitly, but the motivation for his fundamental principle may be the result of the analogy with the mechanical system for which the work depends on the mass of the load but not on the substance of the load.

Carnot argues that for a given difference in temperature the work is smaller at high temperatures and greater at low temperatures so that the dependence on the temperatures is more complex than just being proportional to the difference in temperatures. If we denote by $f\left(\theta_{1}, \theta_{2}\right)$ the function of the temperatures that describes this dependence, then we may write

$$
\frac{w}{q}=f\left(\theta_{1}, \theta_{2}\right)
$$

This equation, valid for a Carnot cycle, complemented by the statement that the function $f$ is universal, that is, it does not depend on the substance of the body undergoing the Carnot cycle, is the expression of the Carnot fundamental principle. Notice that the ratio on left-hand side of equation (1) is the efficiency of an engine operating according to a cycle.

\section{Fundamental laws}

\subsection{Law 1}

According to Kuhn 19], the law of conservation of energy was an example of a simultaneous discovered, announced by several authors between 1842 and 1847, including Mayer [20,21] and Joule 22, 24]. In fact, Mayer and Joule advanced the idea that the dissipation of a certain quantity of work $w$ always results in the generation of the same quantity of heat $q$, and conversely, the disappearance of a certain quantity of heat $q$ gives rise to the same quantity of work $w$, which we write as

$$
w=J q,
$$

where $J$ is a universal constant. This law, which we call Mayer-Joule principle, was interpreted as the conservation of energy, because heat was identified as being related to the work of microscopic particles. However, it should be mentioned that the Mayer-Joule law is a macroscopic law. The law of conservation of energy as advanced by Helmholtz 25], on the other hand, was a microscopic law. It gave support to the Mayer-Joule law but no one derived the macroscopic law from the microscopic law ${ }^{1}$ From now on we use the notation $W$ for the mechanical work and measure heat $Q$ in terms of mechanical unit, which amounts to say that $Q=J q$, and the Mayer-Joule law becomes $W=Q$.

If we consider that a system is undergoing a certain thermodynamic process, the variation of its energy $U$, according to the Mayer-Joule law, is written in the form

$$
\Delta U=Q-W,
$$

where $Q$ is the heat introduced into the system and $W$ is the work performed by the system. In differential form,

$$
d U=d Q-d W
$$

In the case of a mechanical work $d W=p d V$ where $p$ is the pressure and $V$ is the volume of the system. In accordance with the conservation of energy, $\Delta U$ does not depend on the process but just on the final and initial states, which means that $d U$ is an exact differential.

In equation (3), the heat $Q$ is interpreted as work exchanged with the system as much as $W$. The way of distinguishing these two quantities is to say $W$ is the adiabatic work by the introduction of the concept of adiabatic wall. The main property of an adiabatic wall is to forbid the passage of heat. It should be remarked, that this property is not a definition of adiabatic wall, because otherwise we would face a circular reasoning. Adiabatic wall should be understood theoretically as a primitive concept, without the need of definition [26].

\footnotetext{
${ }^{1}$ In fact, the derivation of macroscopic laws from microscopic laws was the aim of the kinetic theory advanced by Clausius, Maxwell and others.
} 


\subsection{Law 2.1}

The Mayer-Joule law was clearly in conflict with Carnot's theory because the latter was based on the conservation of caloric. Joule suggested the abandoning of Carnot's theory [24] but Kelvin disapproved this solution [27]. This conflict was solved by Clausius $[1]$ by observing that Carnot principle, expressed by proposition 3, could be separated into two parts. One of them was the conservation of caloric, implicit in proposition 3 , which could be abandoned. Thus, Clausius retained only the part of Carnot principle expressed by equation (1), which was a consequence of proposition 3, with $q$ interpreted as the heat absorbed in a Carnot cycle, which we denote by $Q_{1}$. Thus, equation (1) becomes

$$
\frac{W}{Q_{1}}=f\left(\theta_{1}, \theta_{2}\right) .
$$

Within Clausius' theory, equation (5), valid for a Carnot cycle, complemented by the statement that the function $f$ is universal, that is, it does not depend on the substance of the body undergoing the Carnot cycle, becomes the expression of the Carnot fundamental principle within Clausius' theory.

In accordance with Mayer-Joule law, Clausius writes

$$
Q_{1}-Q_{2}=W
$$

where $Q_{2}$ is the heat delivered by the working agent. Combining equations (5) and (6), it follows that $Q_{2} / Q_{1}$ is a universal function of $\theta_{1}$ and $\theta_{2}$. By a combination of two Carnot cycles we may conclude that

$$
\frac{Q_{2}}{Q_{1}}=\frac{T\left(\theta_{2}\right)}{T\left(\theta_{1}\right)}
$$

where $T(\theta)$ is a universal function of $\theta$, which we may call absolute temperature. Using the notation $T_{1}=T\left(\theta_{1}\right)$ and $T_{2}=T\left(\theta_{2}\right)$ we may write

$$
\frac{Q_{2}}{Q_{1}}=\frac{T_{2}}{T_{1}}
$$

Employing the convention that heat is positive when it is absorbed by the working agent and negative when it is released, then equation (8) may be writen as $Q_{1} / T_{1}+$ $Q_{2} / T_{2}=0$. The generalization of this equation leads to the result

$$
\oint \frac{d Q}{T}=0
$$

valid for any cycle, which means that $d Q / T$ is an exact differential. Thus there exists a state function $S$, called entropy by Clausius [28], whose differential $d S$ is given by

$$
d S=\frac{d Q}{T} .
$$

It should be remarked that all the equations refer to equilibrium processes, which, in accordance with Clapeyron representation [10] are understood as trajectories in the pressure-volume diagram. Equation with the understanding that $d S$ is an exact differential and that it is valid for a system undergoing an equilibrium process, we call law 2.1. It is the fundamental consequence of the Carnot principle. If we integrate equation 10 along any trajectory, we find

$$
\Delta S=\int \frac{d Q}{T} .
$$

Again, we remark that equation (11) should be understood as valid for an equilibrium process.

Another way of reaching equation (10) was conceived by Carathéodory. Instead of using Carnot principle, he uses a principle stating that 29]: "In any arbitrary neighborhood of a given initial state, there are states that can not be arbitrarily reached by an adiabatic process." In other words, there are states that can only be reached with the exchange of heat. This is the case, for instance, of two states belonging to the same isotherm.

Let us consider an adiabatic work of the general form

$$
d W=\sum_{i} y_{i} d X_{i}
$$

Then, the conservation of energy in differential form, given by equation (4), can be writte as

$$
d Q=d U+\sum_{i} y_{i} d X_{i}
$$

Although $d U$ and $d X_{i}$ are exact differentials, $d Q$ is not in general. Following Born [30], one has to show that there exists an integrating factor of $d Q$. If that is the case, then it suffices to postulate that the reciprocal of this factor is temperature to reach law 2.1 expressed by equation 10 . In the case of more than two term on the right-hand side of (13), there might not exist an integrating factor 30 . However, if the condition given by Carathéodory principle is fulfilled than the integrating factor does exist, as demonstrated by Carathéodory [29], and law 2.1 can also be thought as a consequence of this principle.

\subsection{Law 2.2}

In his first memoir on heat [1], Clausius developed his theory by assuming two fundamental principles. The first was the principle of equivalence of heat and work, advanced by Mayer and Joule and later identified as the conservation of energy, called the first law of thermodynamics. The second was a new principle developed by Clausius, inspired on a fundamental rule advanced by Carnot and related to the production of work in heat machines, which is proposition 2 stated above. According to this new principle of Clausius [1],

Proposition 4: [heat] shows everywhere the tendency to equalize differences of temperature, and thus to pass from a warmer body to a colder one. 
It was rephrased by Kelvin [2], who called the axiom of Clausius, as:

Proposition 5: It is impossible for a self-acting machine, unaided by any external agency, to convey heat from one body to another at a higher temperature,

which became known as the Clausius statement of the second law of thermodynamics. Kelvin also introduced an axiom of his own [2]:

Proposition 6: It is impossible, by means of inanimate material agency, to derive mechanical effect from any portion of matter by cooling it below the temperature of the coldest of the surrounding objects,

which became known as Kelvin statement of the second law. Although the statements have different forms, wrote Kelvin [2], one is a consequence of the other. Later on, Clausius gave his own statement by writing [31:

Proposition 7: Heat can never pass from a colder to a warmer body, if no other related change occurs at the same time.

It can be rephrased in the following terms. Heat flow spontaneously from a hotter to a colder body. A brief statement was also given by Clausius 32 :

Proposition 8: heat cannot by itself pass from a colder to a hotter body.

To be specific, we call proposition 7, Clausius fundamental principle.

From the second law, Clausius establishes the inequality 28,31

$$
\Delta S \geq \int \frac{d Q}{T^{\prime}},
$$

where $\Delta S$ is the variation of the entropy of the system during a process, $d Q$ is the infinitesimal heat exchanged with the environment, being positive when it is introduced into the system, and $T^{\prime}$ is the temperature of the environment, not of the system.

Equation 14, which we call law 2.2, is a direct consequence of the Clausius fundamental principle. To see this, we proceed as follows. We consider a mechanical system consisting of two bodies A and B undergoing certain processes. They are isolated so that the total energy $U+U^{\prime}=U_{0}$ and the total volume $V+V^{\prime}=V_{0}$ are invariant, where unprimed and primed variables refer to bodies $\mathrm{A}$ and $\mathrm{B}$, respectively. To determine the variation of entropy of each body, we may use equilibrium processes because the variation of entropy does not depend on the process but only on the initial and final states, which are assumed to be equilibrium states. Denoting by $\gamma$ and $\gamma^{\prime}$ the equilibrium processes connecting the initial and final states, then the variation of entropy of bodies $\mathrm{A}$ and $\mathrm{B}$ are

$$
\Delta S=\int_{\gamma} \frac{d Q}{T}, \quad \Delta S^{\prime}=\int_{\gamma^{\prime}} \frac{d Q^{\prime}}{T^{\prime}},
$$

where $d Q=d U+p d V$ is the infinitesimal heat received by body $\mathrm{A}$ and $T$ is its temperature, whereas $d Q^{\prime}=$ $d U^{\prime}+p^{\prime} d V^{\prime}$ is the infinitesimal heat received by body $\mathrm{B}$ and $T^{\prime}$ is its temperature.

The process $\gamma^{\prime}$ is understood as a trajectory in space $\left(U^{\prime}, V^{\prime}\right)$, and the process $\gamma$ is understood as a trajectory in space $(U, V)$. We choose the process $\gamma^{\prime}$ to be connect to the process $\gamma$ by means of $U^{\prime}=U_{0}-U$ and $V^{\prime}=V_{0}-V$. The variation of entropy $\Delta S^{\prime}$ is then written as

$$
\Delta S^{\prime}=\int_{\gamma} \frac{d Q^{\prime}}{T^{\prime}}
$$

where the integral is understood as an integral in space $(U, V)$, obtained by the transformation $U^{\prime}=U_{0}-U$ and $V^{\prime}=V_{0}-V$ so that $d U^{\prime}=-d U, d V^{\prime}=-d V$, and $d Q^{\prime}=-d U+p^{\prime} d V$. The total entropy variation is then

$$
\Delta S+\Delta S^{\prime}=\int_{\gamma}\left(\frac{d Q}{T}+\frac{d Q^{\prime}}{T^{\prime}}\right) .
$$

Up to this point, $\gamma$ can be any process connecting the initial and final states. But now, we choose $\gamma$ as composed by two subprocesses. The first one, which we call $\alpha$, is such that $d Q / T+d Q^{\prime} / T^{\prime}=0$ or $\left(1 /-1 / T^{\prime}\right) d U-$ $\left(p / T-p^{\prime} / T^{\prime}\right) d V=0$, which defines a trajectory in space $(U, V)$. Thus along the subprocess $\alpha$ the contribution to the integral (17) vanishes. The second subprocess, which we call $\beta$ is such that $d V^{\prime}=d V=0$ so that $d Q^{\prime}=-d Q$ because in this case $d Q=d U, d Q^{\prime}=d U^{\prime}$, and $d U^{\prime}+d U=0$. Therefore, we may write the total variation of the entropy as

$$
\Delta S+\Delta S^{\prime}=\int_{\beta} d Q\left(\frac{1}{T}-\frac{1}{T^{\prime}}\right) .
$$

Let us suppose that $d Q>0$, that is, the body A receives a certain quantity of heat from the body B. Taking into account that integral $(18)$ is performed keeping the volume of $\mathrm{A}$ invariant, implying that the volume of $\mathrm{B}$ is also invariant, then the only possibility from Clausius fundamental principle is that $T<T^{\prime}$, which can be translated into the inequality $d Q / T-d Q / T^{\prime} \geq 0$. The same inequality holds if the body $\mathrm{A}$ gives heat to the body $\mathrm{B}$ because in this case $d Q<0$, but now $T>T^{\prime}$. We may conclude that the integral in equation 18 is greater or equal to zero so that

$$
\Delta S+\Delta S^{\prime} \geq 0
$$

If we use equation (16) to replace $\Delta S^{\prime}$, we reach the inequality (14).

\section{4. conclusion}

We have argued that the Carnot principle can be considered as a fundamental principle without the need of demonstration, and thus independent of the Clausius fundamental principle. The Carnot principle leads to the 
laws 2.1, represented by equation $(10)$, whereas the Clausius principle leads to law 2.2, represented by the Clausius inequality (14). These two principles, together with the conservation of energy are taken as the fundamental laws of thermodynamics. It is implicit in this approach that temperature is an elementary concept whereas the entropy is a derived concept, that is, entropy is defined by the use of law 2.1, which involves the temperature.

Conceptually, the present approach has certain advantages. For example, we may assume that entropy is an elementary concept holding the property given by equation $(19)$, which is equivalent to law 2.2. The temperature is then considered to be a derived concept, defined by the use of law 2.1. This scheme is in fact used by Callen in his treatment of equilibrium thermodynamics, where temperature is defined by $T=\partial U / \partial S$. It is also implicit in Gibbs equilibrium thermodynamics in which the convexity properties of entropy is a direct consequence of equation 19.

The roots of the second law of thermodynamics, from the theoretical point of view, are found on the memoir of Carnot on heat. We have made an appreciation of this memoir pointing out the understanding of Carnot concerning the production of work by heat, and the ideas that led him to the main principles of his theory of heat. Carnot's theory was based on the conservation of caloric which was found to be in conflict with the Mayer-Joule law on equivalence of work and heat. We have described how this conflict was solved by Clausius. From Clausius fundamental principle, we have shown how to derive the Clausius inequality, the best representation of the second law of thermodynamics.

\section{References}

[1] R. Clausius, Annalen der Physik und Chemie 79, 368, 500 (1850).

[2] W. Thomson, Transactions of the Royal Society of Edinburgh 20, 261 (1853).

[3] A. Sommerfeld, Thermodynamics and Statistical Mechanics (Academic Press, New York, 1956).

[4] E.H. Lieb and J. Yngvason, Physics Report 310, 1 (1999).

[5] J.W. Gibbs, Transactions of the Connecticut Academy 2, 382 (1873).

[6] J.W. Gibbs, Transactions of the Connecticut Academy 3, 108, (1876); 343 (1878).

[7] H.B. Callen, Thermodynamics, (Wiley, New York, 1960).

[8] M.J. Oliveira, Equilibrium Thermodynamics (Springer, Berlin, 2017), 2nd ed.

[9] S. Carnot, Réflexions sur la Puissance Motrice du Feu et sur les Machines propes à Developper cette Puissance (Bachelier, Paris, 1824).

[10] E. Clapeyron, Journal de l'École Royale Polytechnique 14, 153 (1834).

[11] E. Mendoza, in S. Carnot, Reflections on the Motive Power of Fire (Dover, New York, 1960).

[12] W. Thomson, Philosophical Magazine 33, 313 (1848).
[13] D. Mckie and N.H.V. Heathcote, The Discovery of Specific and Latent Heats (Arnold, London, 1935).

[14] R. Fox, The Caloric Theory of Gases (Clarendon Press, Oxford, 1971).

[15] D.S.L. Cardwell, From Watt to Clausius (Cornel Universit Press, Ithaca, 1971).

[16] S.D. Poisson, Théorie Mathématique de la Chaleur (Bachelier, Paris, 1835).

[17] A.L. Lavoisier and P.S. Laplace, Mémoire de l'Académie Royal des Sciences (Imprimerie Royale, Paris, 1783). p. 355.

[18] B.S. Finn, Isis 55, 7 (1964).

[19] T.S. Kuhn, in Critical Problems in the History of Science edited by M. Clagett (University of Wiconsin Press, Madison, 1969), p. 321.

[20] J.R. Mayer, Annalen der Chemie und Pharmacie 42, 233 (1842).

[21] J.R. Mayer, Die organische Bewegung in ihrem Zusammenhange mit dem Stoffwechsel, Ein Beitrag zur Naturkunde (Drechsler, Heilbronn, 1845).

[22] J.P. Joule, Philosophical Magazine 23, 263, 347, 435 (1843).

[23] J.P. Joule, Philosophical Transactions of the Royal Society 140, 61 (1850).

[24] J.P. Joule, Philosophical Magazine 26, 369 (1845).

[25] H. Helmholtz, Über die Erhaltung der Kraft (Reimer, Berlin, 1847).

[26] M.J. Oliveira, Braz. J. Phys. 48, 299 (2018).

[27] W. Thomson, Transactions of the Edinburgh Royal Society 16, 541 (1849).

[28] R. Clausius, Annalen der Physik und Chemie 125, 353 (1865).

[29] C. Carathéodory, Mathematische Annalen 67, 355 (1909).

[30] M. Born, Natural Philosophy of Cause and Chance (Clarendon Press, Oxford, 1949).

[31] R. Clausius, Annalen der Physik und Chemie 93, 481 (1854).

[32] R. Clausius, Annalen der Physik und Chemie 116, 73 (1862). 